series to parallel connected fields in the motor may influence commutation. With such machines as phase-advancers or regulating machines connected to the secondary of induction motors, the switching theory readily explains differences in commutation which have been observed by the authors for different connections of such machines to various external circuits.

The introduction of the switching concept into the commutating problem is of course not necessary when ideal commutating conditions exist. There also are cases which approach the ideal sufficiently to make the consideration of the switching concept unnecessary in practise. Again, where the commutating conditions are not ideal but where the brush shortcircuits several armature coils, their mutual effect may be so predominating that the characteristics of the field and external circuits may have little influence. In general, however, the commutating phenomenon cannot be considered as completely analyzed unless the possibility of the switching phenomenon as influenced by the various circuits has been given due consideration.

\title{
Cathode Drop in Arc Discharges
}

There is no general agreement among physicists concerning the mechanism which produces a law cathode drop with the correspondingly high current density which is characteristic of an electric arc. Herewith is presented a review of prevalent theories regarding this phenomenon, and results of some experiments on arc and glow discharges.

By

\section{S. S. MACKEOWN} ASSOCIATE A.I.E.E.

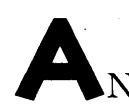

$\mathrm{N}$ ARC is defined as an electrical discharge in a gas or vapor in which the cathode drop is of the order of 10 or 20 volts and the current density to the cathode spot is of the order of hundreds or thousands of amperes per square centimeter. In

Based upon "Cathode Drop in Arcs and Glow Discharges" (No. 31M5) pre sented at the A.I.E.E. Pacific Coast convention, Lake Tahoe, Calif., Aug. $25-28,1931$. order for an arc to exist it is necessary that there be some mechanism for producing electrons at or near the cathode. As to the way in which these electrons are liberated there are three theories as outlined briefly in the following paragraphs.

1. Thermionic emission. This, the oldest theory, assumes that by the bombardment of positive ions the cathode spot is maintained at such a temperature that it is capable of emitting sufficient electrons to maintain the arc. For this to take place temperatures greater than 3,000 deg. cent. would have to exist at the cathode spot. These temperatures actually do exist in the case of the carbon and tungsten arc; but in arcs between less refractory metals such as copper or brass as well as in the mercury arc, no such temperatures are present at the cathode spot. In such cases thermionic emission cannot account for the liberation of sufficient electrons to maintain the arc.

2. A more recent theory is that positive ions are present close to the cathode in such large numbers that they produce an electric field sufficiently large to free electrons from the cathode by the attraction of the field alone. Calculations show that electric fields of the order of $10^{6}$ volts per $\mathrm{cm}$. exist at the cathode in the case of both copper and mercury arcs; these fields are large enough to supply the electrons necessary for maintaining the arc.

3. A third theory proposes that the production of electrons is due to thermal ionization of the gas near the cathode. In order that there should be any appreciable thermal ionization in the gas, the temperature of the gas must exceed 4,000 deg. cent.; it is doubtful if such a high temperature obtains.

It seems probable therefore in the case of arcs between highly refracting electrodes, such as carbon and tungsten, that electrons are emitted from the cathode by thermionic action. In the case of arcs between less refractory electrodes such as copper or brass, electrons probably are emitted from the cath-

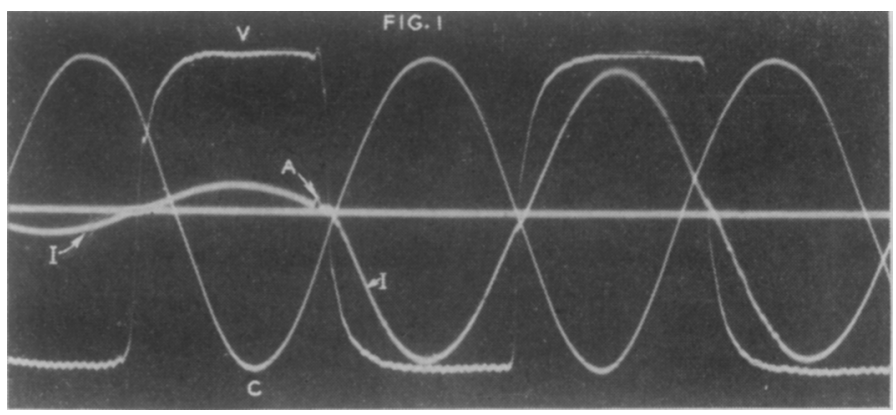

Fig. 1. Current and voltage relations in the abnormal cathode drop; at point $A$ the current was increased suddenly from 52 to 320 milliamperes

ode by the action of the intense electric field existing at the cathode surface.

\section{Normal Cathode Drop in the Glow Discharge}

In the glow discharge a much higher cathode drop exists than in the arc. If the current density to the cathode is below a certain definite value, the cathode drop is constant and is spoken of as the normal cathode drop. As the current density to the cathode is increased further the magnitude of the cathode drop increases, and the region over which it extends decreases. When the cathode drop increases with increasing current density it is spoken of as the abnormal cathode drop.

In the glow discharge as in the arc there must be some mechanism present to produce electrons at or 
near the cathode; the following theories have been proposed:

1. Positive ion bombardment of the cathode. It is known that electrons may be liberated by positive ion bombardment of $/$ a metal surface; however, when the normal cathode drop obtains it is doubtful if the positive ions hit the cathode with sufficient energy to produce any appreciable ionization; but when the cathode drop is abnormal, the positive ions possess much more energy, and under these conditions this mechanism may be the most important one.

2. Production of electrons by collision of positive ions with neutral gas molecules in the region of the cathode drop. In general this process occurs only when the positive ions possess energy greater than in the normal cathode drop.

3. Production of electrons from the cathode by photoelectric effect. It is believed generally that this process is inadequate to account for the required number of electrons necessary to maintain the discharge.

4. Production of electrons by metastable atoms arriving at the cathode. Because one of the electrons is in a high-energy level, these metastable atoms have enough potential energy to free electrons from the cathode. It is probable that in the case of the normal cathode drop this mechanism is important, especially when the rare gases such as helium, neon, or others are used.

Unfortunately, so far none of these theories has been developed so as to obtain quantitative agreement with experimental results. It is possible that two or more of these processes may take place simultaneously; also in some cases one of them may be most important, whereas in other cases another may be more prominent.

\section{Abnormal Cathode Drop}

As the current to the cathode of a discharge tube is increased in the region of the abnormal cathode drop, the magnitude of the cathode drop increases and the distance from the cathode in which the cathode drop exists decreases. Both of these effects cause an increase in the electric field existing in the cathode drop. It is quite certain that as the current is increased, a greater portion of the current to the cathode is due to electrons leaving the cathode.

For the past several years considerable experimental work has been done on the abnormal cathode

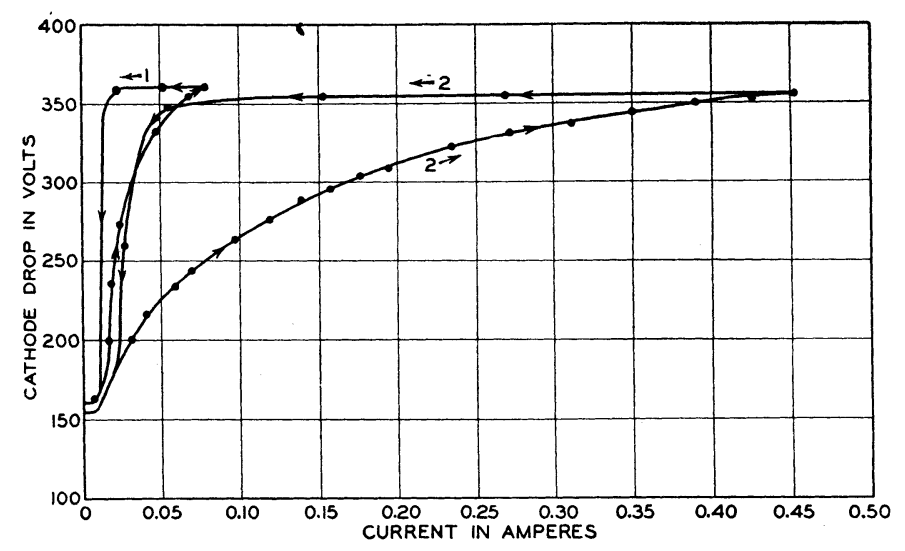

Fig. 2. Voltage of Fig 1 plotted against current

drop; the results of a few of the experiments are presented here. Preliminary measurements of the cathode drop, especially when the current to the cathode was large compared to that required to just cover the cathode with the cathode glow, gave quite inconsistent results. The first measurements were made with direct current, but soon it was found better to use alternating current and measure the cathode drop with an oscillograph.

In Fig. 1 is an oscillogram which shows the relation between current and voltage in the abnormal cathode drop. Curve $I$ represents the current through the tube, $V$ the voltage across the tube, and curve $c$ a calibration curve which in this discussion may be disregarded. At point $A$ a resistance in series with the tube was short-circuited so that the current was increased from 52 to 320 milliamperes

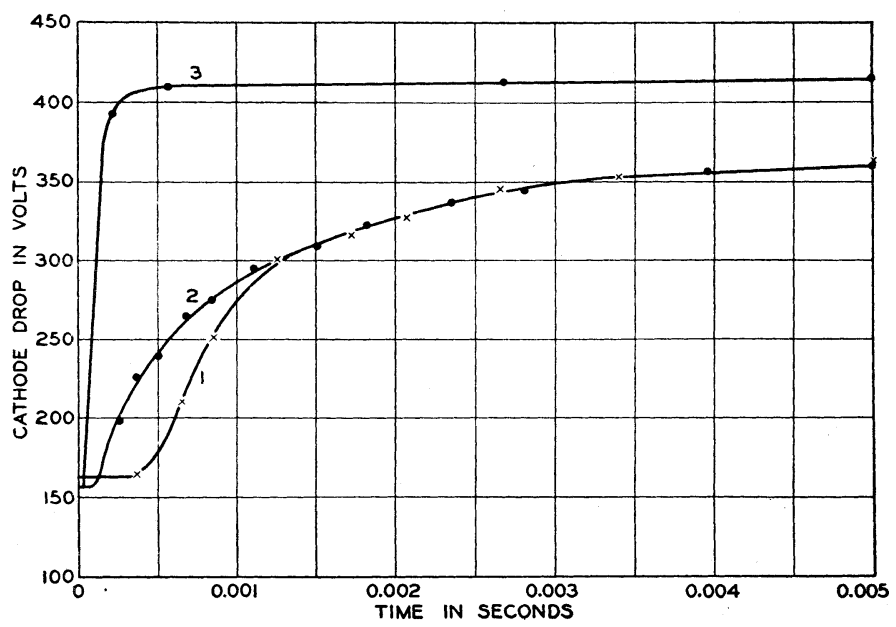

Fig. 3. Voltage of Fig. 1 plotted against time

(r.m.s.). It is apparent at once that this change in current has only a small effect upon the voltage curve.

In Fig. 2 the data from Fig. 1 are plotted with voltage and current as ordinates. This curve shows that the cathode drop is less for increasing values of current than it is for decreasing values. In Fig. 3 the data from Fig. 1 are plotted with cathode drop and time as ordinates. Curve 1 represents the data for increasing current when the effective value of the current is 52 milliamperes; curve 2 for a current of 320 milliamperes, and curve 3 for a current of $4 \mathrm{am}$ peres. The difference between curves 1 and 2 for low values of time can be explained by the fact that the abnormal cathode drop occurs at an earlier time when the current is high than when it is low. If allowance for this is made it is apparent that the cathode drop requires approximately the same time to build up in the two cases where the current is 52 and 320 milliamperes, respectively. Curve 3 , however, shows that the cathode drop builds up much more rapidly when the current is high; this difference is explained as follows: In the case where the current is low, the cathode drop is formed by the positive ions from the main body of the gas congregating near the cathode, a relatively slow process. When the current density to the cathode is high, the cathode drop is due to the ionization of gas molecules near the cathode caused by electrons leaving the cathode and by the rapid dispersion of electrons away from the neighborhood of the cathode. Since electrons move hundreds of times more rapidly than positive ions, 
this latter process takes place much more rapidly. This leads to the conclusion that when the current density is high, a large part of the current to the cathode is carried by electrons leaving the cathode.

\section{Transition From Glow to ARC}

The transition from a glow discharge to an arc is illustrated by Fig. 4. When this transition occurs, the voltage (curve $V$ ) shows a sudden decrease which corresponds to the decrease in cathode drop. Current through the tube is represented by curve $I$; its effective value was 4 amperes. The fact that sometimes an arc is formed and sometimes not, probably is due to the fact that the surface of the cathode is constantly changing because of the rapid sputtering or disintegration that occurs in the glow discharge with such high current densities.

A consideration of results from several oscillograms similar to Fig. 4 leads to the following explanation of the process by which a glow discharge changes to an arc: As the current to the cathode is

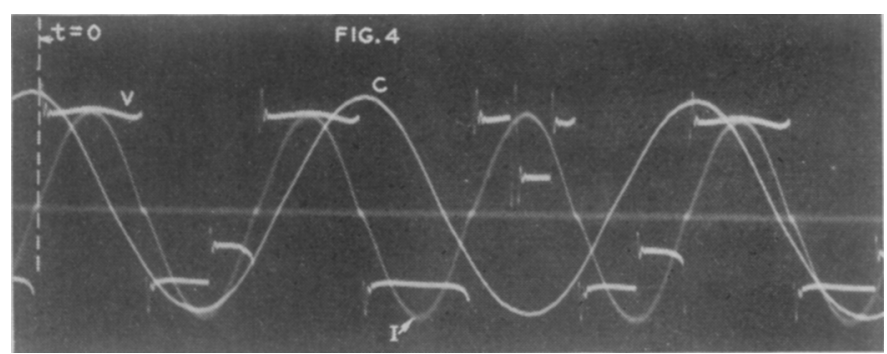

Fig. 4. Current and voltage relations during transition from glow to arc

increased in the glow discharge, a larger portion of current is due to electrons leaving the cathode; these electrons are not emitted uniformly from the cathode surface, but come primarily from small portions of the cathode, which because of some impurity are especially active. These electrons produce ionization in the region of the cathode drop, this ionization being greatest in the vicinity of the active spots. Due to their high mobility, the electrons leave this region much more rapidly than do the positive ions. A high space charge results, increasing the electric field in the vicinity of these active spots as well as the number of electrons emitted therefrom. This process is cumulative and proceeds rapidly until the electric field near one of the spots increases to an amount sufficient to produce electrons from the cathode by the attractive force of the field alone. When this occurs, an arc is formed with its low cathode drop and extremely high current density to the cathode spot.

This explanation is based upon arcs at low pressure; it is probable, however, that the same process is effective at atmospheric pressure, and occurs every time the current in an a-c. arc passes through zero, except when the electrodes are so refractory that they can support a high enough temperature to produce a large electron current by thermionic emission.
Transient Oscillations in Coupled Windings

\begin{abstract}
A mathematical study of the oscillations occurring in a pair of mutually coupled windings, the primary and secondary of a transformer, when one of the terminals is subjected to the impact of a traveling wave, has been completed. The general conclusions reached in this study are summarized in the following article.
\end{abstract}

General Elec. Co.,
Pittsfield, Mass.

$$
\begin{aligned}
& \text { L. V. BEWLEY } \\
& \text { ASSOCIATE A.I.E.E. }
\end{aligned}
$$

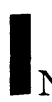

N THE PAST, the mathematical analysis of transient oscillations in transformer windings has been based upon a single winding having self-inductance and mutual inductance between its turns, capacitances along the stack and to ground, and in one instance, resistances to represent the losses. Such a circuit ignores the presence of secondary circuits, but, strangely enough, proves quite adequate to describe the internal high-frequency transients of the winding under consideration, not only qualitatively, but quantitatively as well.

Cathode ray oscillograms, however, do show some difference in the characteristics of the oscillations, depending upon the terminal connections of the secondary circuits. This is particularly true in the case of an isolated neutral primary winding, where a change from an open-circuited to a short-circuited secondary may bring about considerable changes in the frequencies of oscillations. This shift of frequencies has been attributed loosely to a change in the effective inductance of the circuit, brought about by closing the secondary circuit; just as the natural frequency of a simple lumped inductance-capacitance series circuit is changed if the inductance is reduced by short-circuiting a coupled winding. In addition to the above limitation of the single winding theory of transformer oscillations, there was nothing in the equations relative to the internal oscillations of the secondary circuit.

The complete idealized circuit of a two-winding transformer is shown in Fig. 1. The primary winding, on which the traveling wave is assumed to impinge, is designated as winding 1 , and all associated voltages and currents have the corresponding subscript as $e_{1}, i_{e 1}, i_{k 1}$, and $i_{\mathrm{L} 1}$. The secondary winding is

Based upon "Transient Oscillations of Mutually Coupled Windings" (No, 32-4) presented at the A.I.E.E. winter convention, New York, N. Y., Jan. 25-29, 1932. 